\title{
ENFERMEDAD TROFOBLASTICA
}

\section{EN EL HOSPITAL SAN JUAN DE DIOS. CUCUTA}

\author{
Dr. Alberto Duarte-Contreras M.D.*
}

Dr. Rafael Darío Rolón-Duarte M.D.**

Dr. Manuel José Palau-C. M.D.**

Nos proponemos hacer en la presente revisión un análisis estadístico de 53 casos de enfermedad trofoblástica observados en el Departamento Gíneco Obstétrico del Hospital San Juan de Dios, de Cúcuta, estudiar su potencialidad maligna y valorar los métodos de diagnóstico, tratamiento y control posterior comparando nuestros hallazgos con lo observado por investigadores tanto nacionales como extranjeros; y al mismo tiempo presentar la bibliografía nacional sobre este tópico $(1,10,19,20,22,27,33$, $37,43,48,49,53,54,55,59)$.

\section{Material y Métodos}

El personal de gestantes que acuden a nuestro servicio no es seleccionado desde el punto de vista obstétrico y pertenece en totalidad a la clase social económica menos favorecida ,cuyo aporte calórico y nutricional está por debajo de los requerimientos mínimos; y dada nuestra localización proceden casi todas de climas cálidos con temperatura media entre 26 y 32 grados centígrados.

Nuestro estudio abarca 8 años (1? de enero de 1962 a 31 de diciembre de 1969). En este lapso atendimos un total de 28.274 gestantes, 17.259 partos y 3.815 abortos.

\section{Incidencia}

Una de nuestras pacientes ingresó con coriocarcinoma y metástasis múltiples; 52 llegaron con embarazo molar, lo que nos da una mola por cada 543 embarazos $(0,18 \%)$, una por cada 324 partos, una por cada 73 abortos.

En la literatura nacional Vergara Támara (54) al analizar pacientes que pertenecen a la "blenda racial mestiza, mulatas y algunas blancas, las menos" de Bogotá y sus alrededores, y de situación económica de clase media, encuentra una mola por cada 508 embarazos. Acosta Bendek (1) estudia pacientes mestizas de la costa norte, de bajo grupo social y encuentra una por cada 311 embarazos. Y Valencia y colaboradores (53) estudian gestantes de clima frío $y$

* Médico Jefe del Departamento Gíneco Obstétrico del Hospital San Juan de Dios, Cúcuta, Colombia.

* Médico Adjunto del Departamento Gíneco Obstétrico del Hospital San Juan de Dios, Cúcuta. 
CUADRO 1

MOLA HIDATIFORME. - INCIDENCIA

\begin{tabular}{|c|c|c|c|c|}
\hline Autor & Una & por & $\mathrm{N}^{0}$ partos & Nación \\
\hline Novak (42) & 1 & por & 2.500 & U. S. A. \\
\hline Hertig (29) & & & 2.000 & U. S. A. \\
\hline Jeffcoate (31) & & & 2.000 & Inglaterra \\
\hline Alter (7) & & & 1.720 & U. S. A. \\
\hline Demers (17) & & & 1.450 & Canadá \\
\hline De Snoo (18) & & & 1.200 & Holanda \\
\hline Westerhout (58) & & & 1.115 & U. S. A. \\
\hline Fernández (24) & & & 1.071 & Brasil \\
\hline Meyer (41) & & & 909 & Alemania \\
\hline Pinedo (46) & & & 834 & Venezuela \\
\hline Coppelson (16) & & & 800 & Australia \\
\hline Aramburu (9) & & & 670 & Guatemala \\
\hline McGregor (39) & ' & & 609 & México \\
\hline Cabrera (12) & & & 609 & Chile \\
\hline Kink (35) & & . & 530 & China \\
\hline Vergara (54) & & & 508 & COL.OMBIA, Bogotá \\
\hline Brindeau (11) & & & 500 & Francia \\
\hline Fernández D. (26) & & & 400 & México \\
\hline Karzavina $(34)$ & & & 330 & Rusia \\
\hline Acosta-Bendek (1) & & & 311 & COLOMBIA, Barranquilla \\
\hline Poen (47) & & & 229 & Indonesia \\
\hline Márquez (38) & & & 200 & México \\
\hline Acotsa-Sison (4) & & & 173 & Filipinas \\
\hline Valencia (53) & & & 155 & COLOMBIA, Manizales \\
\hline Wei-Ping (57) & & & 82 & Formosa \\
\hline Presente Estudio & & & 543 & COLOMBIA, Cúcuta \\
\hline
\end{tabular}

medio $(84,6 \%$ procedentes de Manizales, clima frío, y $15,4 \%$ de poblaciones cafeteras de Caldas, clima medio) y hallan una por cada $155 \mathrm{em}-$ barazos, cifra ésta superior a la citada en 1951 por Acosta Sison en Filipinas (4) (una por cada 173 gestantes) y apenas superada por WeiPing de Formosa (57) (una por cada 82 embarazos).

Nuestra incidencia de una por cada 543 embarazos corresponde a gestantes mestizas, de clima cálido, procedentes en un $61 \%$ de región rural selvática mas no de raza indígena. $\mathrm{Si}$ bien la cifra que encontramos es alta, no es tan alarmante como la hallada por Valencia y colaboradores en el Departamento de Caldas. Y si comparamos con estadísticas extranjeras nuestra inncidencia es interme- dia entre las cifras reportadas por McGregor de México (39), Cabrera de Chile (12) y por Kinnk de China (35) y Brindeau de Francia (11).

En lo que respecta a la degeneración maligna de la enfermedad trofoblástica, Vergara informa de un caso por 5.084 embarazos; Acosta Bendek reporta un coriocarcinoma por cada 4.363 embarazos y Valencia uno por cada 2.832 gestantes. Nosotros encontramos cuatro casos de coriocarcinoma, o sea uno por cada 7.068 embarazos.

Si rveisamos la literatura universal veremos datos muy disímiles con incidencias que van desde un coriocarcinoma por cada 77.592 partos hasta uno por cada 476 partos y desde uno por cada 44.000 embarazos 


\section{CUADRO 2}

\section{CORIOCARCINOMA. - INCIDENCIA}

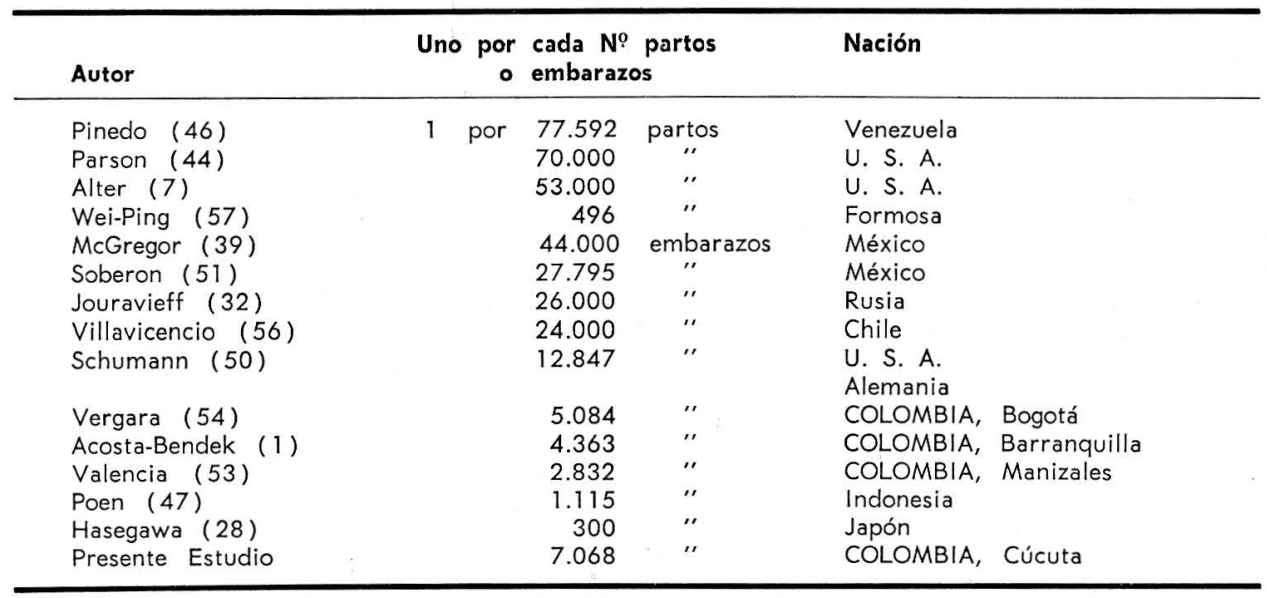

hasta uno por cada 300 embarazos con cifras intermedias que podemos analizar en el Cuadro 2.

Nos queda muy en claro que la incidencia del coriocarcinoma en cuatro regiones estudiadas de Colombia es muy alta, siendo superada únicamente por la de los países de Oceanía y los del extremo oriental asiático.

Edad de las pacientes

\section{CUADRO 3}

MOLA HIDATIFORME EDAD DE LAS PACIENTES

\begin{tabular}{ccccc}
\hline \multicolumn{2}{c}{ Edad } & No & Casos & $\%$ \\
\hline 14 & a & 19 & 10 & 19,22 \\
20 & a & 29 & 24 & 46,16 \\
30 & a & 39 & 15 & 28,85 \\
40 & a & 49 & 3 & 5,77 \\
\hline
\end{tabular}

En nuestra casuística la paciente de menor edad fue una primigesta de 14 años y la de mayor edad una gesta 17 de 49 años. El más alto por- centaje lo encontramos en la segunda década $(46,16 \%)$ lo que se explica por el mayor número de gestaciones en este período. Todas nuestras cifras en relación con la edad, coinciden con los hallazgos de la mayoría de los investigadores que hemos consultado.

En lo que respecta al coriocarcinoma, una de nuestras pacientes tenía 34 años, dos tenían 35 años y la restante 36 años o sea estaban todas entre la tercera y la cuarta década de la vida lo que está acorde con la edad promedio de mayor frecuencia encontrada por algunos autores en la degeneración maligna $(1,38,57)$, si bien otros reportan una mayor incidencia en la segunda y tercera década (47).

Observamos como máxima paridad una paciente G. 17 P. 16. La mayor frecuencia en nuestra serie correspondió a pacientes $G .2$ a $G$. 6 $(42,31 \%)$. $Y$ es muy notorio el hecho de que el número de molas en las grandes multíparas $(30,77 \%)$ sea 


\section{Pardiad}

\section{CUADRO 4}

MOLA HIDATIFORME. - PARIDAD

\begin{tabular}{lccc}
\hline Paridad & N $^{\mathbf{0}}$ Casos & $\%$ \\
\hline Para VII a Para XVI & 16 & 30,77 \\
Nulíparas & 8 & 15,38 \\
Primíparas & 6 & 11,54 \\
Grandes multíparas: & & \\
Para II a VI & 22 & 42,31 \\
\hline
\end{tabular}

el doble del que hallamos en las nulíparas o primigestas $(15,38 \%)$ y mayor aún del que observamos en las primíparas $(11,54 \%)$.

\section{Antecedentes Obstétricos}

De las 52 pacientes con mola, 17 tuvieron antecedente de aborto: 14 con un aborto anterior, dos con dos abortos y una con tres abortos. Una gestante tenía antecedente de desprendimiento prematuro de placenta normoinserta en el embarazo inmediatamente anterior. Encontramos una mola de repetición. $Y$ en lo que respecta al coriocarcinoma tres casos fueron inmediatamente precedidos por aborto molar y uno por un parto eutócico dos años antes: esta paciente ingresó con metástasis a pulmón y a vagina.

La edad promedio de nuestros embarazos molares fue de 18 semanas. Si dividimos nuestra casuística en tres grupos, observamos los siguientes porcentajes: el $28,85 \%$ de las molas tenían de 8 a 15 semanas, el $40,38 \%$ de 16 a 20 semanas y el $30,77 \%$ de 21 a 25 semanas. O sea: hay una mayor frecuencia en el grupo de 16 a 20 semanas; pero en las comprendidas entre 20 y 25 semanas nuestra incidencia es superior a la que anotan otros autores nacionales $(1,58)$ y extranjeros (38) para este mismo grupo de edad clel emba-
Edad del Embarazo molar al ingreso

\section{CUADRO 5}

EMBARAZO MOLAR EDAD DEL EMBBARAZO

\begin{tabular}{|c|c|c|c|c|}
\hline Semazas & $N^{0}$ & Casos & $\%$ & Total \\
\hline 8 & & 1 & 1,92 & \\
\hline 10 & & 3 & 5,77 & \\
\hline 11 & & 2 & 3,85 & \\
\hline 13 & & 2 & 3,85 & \\
\hline 14 & & 4 & 7,69 & 15 \\
\hline 15 & & 3 & 5,77 & $(28,85 \%)$ \\
\hline 16 & & 2 & 3,85 & \\
\hline 17 & & 4 & 7,69 & \\
\hline 18 & & 8 & 15,38 & \\
\hline 19 & & 2 & 3,85 & 21 \\
\hline 20 & & 5 & 9,61 & $(40,38 \%)$ \\
\hline 21 & & 2 & 3,85 & \\
\hline 22 & & 4 & 7,69 & \\
\hline 23 & & 4 & 7,69 & \\
\hline 24 & & 2 & 3,85 & 16 \\
\hline 25 & & 4 & 7,69 & $(30,77 \%)$ \\
\hline
\end{tabular}

razo; lo que podríamos explicar por la procedencia de la mayoría de nuestras pacientes con mola, región selvática $(61 \%)$, su bajo nivel cultural y la dificultad en el transporte, factores todos causantes de consulta tardía, y de ingreso en estado general muy deteriorado.

\section{Patología concomitante}

CUADRO 6

MOLA HIDATIFORME PATOLOGIA CONCOMITANTE

\begin{tabular}{|c|c|c|c|}
\hline Entidad nosológica & No & pacientes & $\%$ \\
\hline Paludismo & & 6 & 11,54 \\
\hline Toxemia & & 4 & 7,68 \\
\hline Hipertensión arterial & & 2 & 3,85 \\
\hline Shock hipovolémico & & 2 & 3,85 \\
\hline TBC pulmonar & & 1 & 1,92 \\
\hline
\end{tabular}

El alto índice de pacientes palúdicas $(11,54 \%)$ se explica por la procedencia, ya que venían de las sel- 
vas del Catatumbo y del Sarare, donde el paludismo es aún endémico muy a pesar de las campañas de erradicación adelantadas por las autoridades sanitarias.

El hallazgo de $7,68 \%$ de gestantes con Toxemia es muy bajo si lo comparamos con los datos suministrados por Valencia (16,5\%) (53), por Pernia de Venezuela (20\%) (45), por Alter de U.S.A. (20,4\%) (7), por Acosta Bendek (22,9\%) (1) y por Márquez de México (35\%) (38). Todas nuestras toxémicas corresponden al grupo de 20 a 25 semanas de gestación. La Toxemia como complicación del embarazo ocurre generalmente después de la semana 24; su hallazgo a edad más temprana nos obliga a indagar el embarazo molar entre otras entidades.

No encontramos eclampsia en nuestra serie como lo han reportado Castelazo (14) y Estrada Ocampo (23) en México y Cardoso (13) en Brasil.

Es oportuno recordar que según los estudios de Sánchez-Torres y colaboradores (49) la severidad de las lesiones renales en los casos de Toxemia y mola concomitante no están en relación con el grado de proliferación o de invasión o de malignidad del tejicio trofoblástico sino acorde con la severidad de las manifestaciones clínicas de la Toxemia.

Una de nuestras pacientes presentaba un proceso avanzado de tuberculosis pulmonar. Acosta Sison advierte sobre la influencia adversa de la tuberculosis pulmonar en el embarazo molar $(5,6)$; pero con todo y tener nuestra paciente una tuberculosis avanzada, presentaba una mola clasificada como aparentemente benigna.
Los dos casos de shock hipovolémico nos indican claramente el estado en que llegan algunas de nuestras pacientes al servicio.

\section{Síntomas}

Todas consultaron por hemorragia: $19(36,5 \%)$ por hemorragia escasa y $33(63,5 \%)$ por hemorragia abundante, tres de las cuales $(5,9 \%)$ expulsaban vesículas.

El tamaño uterino fue sensiblemente igual al que corresponde a un embarazo normal en $16(30,7 \%)$ y mayor en las 36 restantes $(69,3 \%)$.

\section{Diagnóstico}

En 33 casos $(63,4 \%)$ el diagnóstico presuntivo previo al vaciamiento fue correcto; $y$ en los 19 restantes $(36,6 \%)$ fue errado habiéndose diagnosticado placenta previa en un caso, desprendimiento prematuro de placenta normoinserta en otro y aborto incompleto en $17(32,8 \%)$. Luego se estableció en estas pacientes el diagnóstico de mola por el aspecto macroscópico del material evacuado y se confirmó por el estudio anatomopatológico.

Al revisar las historias encontramos que únicamente a 15 pacientes se les practicó "cuantificación" de gonadotrofinas coriónicas antes del vaciamiento.

Estudiamos 5 pacientes con 26 urocitogramas y encontramos en 3 de ellas aumento de las células cianófilas profundas; en una hubo aumento de la eosinofilia y pignosis $y$ en la quinta la citología correspondió a un embarazo normal (20).

A cinco pacientes se les practicó examen radiológico, habiendo sido negativo para partes fetales. $Y$ a 8 se les hizo estudio radiológico de tó- 
rax como parte del control posterior habiéndose encontrado metástasis en una de ellas.

\section{Anatomía Patológica}

En la totalidad de nuestros casos confirmamos el diagnóstico con estudio histopatológico. Según la clasificación de Hertig y Mansell (25) podemos agrupar nuestras molas de la siguiente manera:

Grado I aparentemente benignas $24(46,15 \%)$.

Grado II potencialmente malignas $21(40,39 \%)$.

Grado III aparentemente malignas $7(13,46 \%)$.

Dos pacientes presentaron mola invasora (corioadenoma destruens).

En 6 pacientes $(11,54 \%)$ encontramos quistes luteínicos bilaterales, uno de ellos complicado con ruptura y hemorragia severa post histerectomía, lo que nos obligó a nueva intervención quirúrgica y extirpación del quiste sangrante.

\section{Tratamiento}

A 50 pacientes les practicamos legrado instrumental, con inducción previa de ocitocina a tres de ellas. Ocho pacientes requirieron un nuevo legrado. A una le hicimos histerectomía y legrado inmediato y a 8 les practicamos histerectomía posterior al legrado. A una paciente que ingresó con coriocarcinoma y metástasis a distancia la tratamos con terapia citostática (Metrotexate).

Las 8 histerectomías se practicaron: una por atonía uterina y hemorragia, dos por corioadenoma destruens, dos en grandes multíparas imposibles de controlar por su procedencia y tres por coriocarcinoma.
Reintervenimos una paciente para extirpar un quiste luteínico sangrante.

\section{Control posterior}

En general el control de nuestras pacientes ha sido deficiente debido a su procedencia y a su bajo nivel cultural. Solamente 24 pacientes asistieron a control un mes después del legrado $(49 \%)$, y de éstas 17 dieron gonadotrofinas negativas y 7 gonadotrofinas positivas.

El control de dos meses se pudo practicar únicamente en 10 pacientes: en 4 de ellas persistían títulos altos de gonadotrofinas (hasta 200.000) más sangrado y subinvolución uterina; se les practicó nuevo legrado y en 3 el diagnóstico histopatológico fue de coriocarcinoma.

Unicamente cuatro pacientes asistieron a control de tres meses, todas con gonadotrofinas negativas.

Las tres pacientes histerectomizadas por coriocarcinoma, si bien se dieron de alta en aceptables condiciones generales no volvieron a control alguno muy a pesar de que se les dieron instrucciones precisas y oportunas.

\section{Evolución obstétrica posterior}

Conocemos la evolución obstétrica de 17 pacientes: cuatro fueron tratadas por aborto incompleto entre uno y tres años después del aborto molar; 13 presentaron embarazo con parto eutósico a término entre uno y ocho años; siete pacientes tuvieron un parto, dos pacientes dos partos, tres pacientes tres partos y una paciente cuatro partos.

\section{Mortalidad}

La mortalidad de nuestra serie fue de tres pacientes, lo que repre- 
senta el 5,77\%. Dos murieron por shock hipovolémico irreversible y una por coriocarcinoma con metástasiss múltiples y aplasia medular como complicación del tratamiento con Metrothexate.

\section{Comentarios}

Vemos que la incidencia de la enfermedad trofoblástica entre nosotros es alta, pero desconocemos la magnitud del problema colombiano ya que hasta ahora solamente se ha estudiado en cuatro zonas del país; ello hace indispensable la elaboración de una encuesta epidemiológica a nivel nacional.

Practicamos el urocitograma en estas pacientes únicamente con fines investigativos. Pero si analizamos nuestros hallazgos (20) y los comparamos con los datos suministrados por invesíigadores extranjeros (8, 36,52 ) vemos que la citología urinaria en casos de mola da cuadros muy divergentes que van desde el normal hasta serias modificaciones eosinófilas y cianófilas, lo que bien podría deberse a la edad evolutiva de la mola y a sus características histológicas.

El escaso número de pacientes controladas posteriormente no se debe a deficiencia de nuestra institución sino a la imposibilidad física de poder localizar pacientes dada su procedencia, a más dal bajo nivel cultural y económico de todas ellas; pero creemos que con una mejor motivación tal vez pudiéramos alcanzar en el futuro una mayor asistencia al control.

Queremos recalcar que de las 10 pacientes que asistieron al segundo control, cuatro lo hicieron por persistencia del sangrado y en tres de ellas encontramos coriocarcinoma.
Aun cuando nuestra cifra de error diagnóstico es baja en relación con la de otros autores, insistimos en que se debe tener más en mente la posibilidad diagnóstica de mola.

Ante un embarazo molar en pacientes de bajo nivel cultural y de difícil control, somos partidarios de practicar la histerectomía siempre y cuando hayan completado el número deseado de hijos.

\section{Resumen y Conclusiones}

Se estudian 53 casos de enfermedad trofoblástica del Hospital San Juan de Dios, Cúcuta, Colombia, en pacientes de bajo nivel socio económico, de clima cálido.

Encontramos una mola por cada 543 embarazos, una por cada 324 partos y una por cada 73 abortos; y un coriocarcinoma por cada 7.068 embarazos.

Se analiza la edad de las pacientes y su paridad: se obtienen resultados similares a los que presentan la mayoría de los autores. En lo que respecta a la edad del embarazo encontramos la mayor incidencia entre 16 y 20 semanas, pero el grupo de 20 a 25 semanas es sensiblemente mayor al encontrado por otros investigadores.

Nuestro diagnóstico clínico fue errado en el $36,6 \%$ de los casos. Insistimos en la necesidad de tener más en mente la posibilidad diagnóstica de enfermedad trofoblástica.

Seguimos la clasificación histopatológica de Hertig y Mansell con el siguiente resultado: grado I el $46,15 \%$, grado II el $40,49 \%$ y grado III el $13,46 \%$. Tuvimos dos casos de corioadenoma destruens, y uno de mola de repetición. En 6 pacientes 
encontramos quistes luteínicos bilaterales.

Como tratamiento practicamos un legrado a 42 pacientes, dos legrados a 8, histerectomía a una (por diagnóscito errado) y a 8 histerectomía post legrado. Una paciente con coriocarcinoma y metástasis a distancia fue tratada con Metrothexate.

Por regla general el control fue deficiente debido a la no asistencia de las pacientes. Se impone una mayor motivación por parte del personal científico hacia ellas.

En pacientes de difícil o imposible control y que hayan dado su cuota anhelada de hijos, practicamos la histerectomía.

Nuestra mortalidad fue del 5,77\%.

Recopilamos la literatura nacional.

\section{Summary}

We study 5.3 cases of trophoblastic disease in 28.274 pregnancies of low socioeconomical estratus, at the Hospital San Juan de Dios, Cúcuta, Colombia.

The frequency was of one mole for every 543 pregnancies, one for every 324 deliveries and one for every 73 abortions; and the incidence of the choriocarcinoma was one for every 7.068 pregnancies. An analysis is made of the age and parity of the patients, and of the age of the pregnancy.

Histopathologic grading of the hidatidiform moles acording with Hertig and Mansell clasification was: grade I, $46.15 \%$; grade II, 40,49\% and grade $111,13,46 \%$. We faund two cases of choriocarcinoma, and one case of succesive mole.
We made single couretage in 42 patients, two couretage in 8 , histerotomia and couretage in one, and 8 histerectomies. One patient with choriocarcinoma recibed Metrothexate.

The control was deficient because poor colaboration of patients.

The incidence of mortality was $5.77 \%$.

\section{BIBLIOGRAFIA}

1 ACOSTA-BENDEK, E. Mola hidatiforme. Comentarios a propósito de 168 observaciones. Rev. Colom. Obst. Gin. 15: 233, 1964.

2 ACOSTA-BENDEK, E. Tumores del trofoblasto. A propósito de doce casos de corioepitelioma y dos de corioadenoma destruens. Rev. Colomb. Obst. Gin. 15: 405, 1964.

3 ACOSTA-BENDEK, E. Nuevo instrumento. Cucharilla molar. Rev. Colomb. Obst. Gin. 19: 448, 1968.

4 ACOSTA-SISON, H. E. and PANLILIO, B. H. Statistical study of 177 cases of hydatiform mole admitted to the Philippine General Hospital from april 6, 1945 to december 31, 1950. J. Philipp. Med. Ass. 27: 652, 1951.

5 ACOSTA-SISON, $H$. Changing attitudes in the management of hydatidiform mole. Am. J. Obst. and Gynec. 88: 634, 1964.

6 ACOSTA-SISON, H. Influence of pulmonary Tuberculosis of Hydatidiform mole. Obst. and Gynec. 20: 103, 1962.

7 ALTER, N. M. and COSGROVE, S. A. Hydatiform mole: Practical considerations. Obst. and Gynec. 5: 755, 1955.

8 ALVAREZ-BRAVO, A., GONZALEZ-RAMOS, H., GUTIERREZ-MURILLO, E. Y DOSAL DE LA VEGA, M. La citología vaginal y la citogloía del sedimento urinario en el embarazo. Ginec. Obstet. Méx. 12: 73, 1957.

9 ARAMBURU, B. G. Mola hidatiforme en el centro Materno Infantil del Instituto Guatemalteco de Seguridad Social. Mem. III Cong. Lat. Amer. Ginec. Obstet. 2: 576 , 1958.

10 BOTERO, I. A. Tratamiento curativo de la mola hidatiforme. Gaceta Médica. 4: 125, 1915. 
11 BRINDEAU, A., HINGLAIS, H. et HINGLAIS, M. La mole hydatiforme. Bull. Féd. Gynec. Obstet. Franc. 4: 3, 1952.

12 CABRERA, $H$. Algunas consideracoines sobre mola hidatiforme. Bol. Soc. Chile. Obstet. Ginec. 11: 107,1946.

13 CARDOSO, C. y MONTARGIL, E. Dos casos de eclampsia en gravidez molar. Rev. Clin. do Inst. Maternal. 40: 23, 1960.

14 CASTELAZO-AYALA, L., MAQUEO, T. M. y BARRIOS, P. L. Mola hidatiforme: estudio de 40 casos con presentación de uno complicado con eclampsia y nódulo vaginal y otro de mola de repetición. Ginec. Obste. Méx. 17: 383, 1962.

15 CASTILlO-VEGA, E. Rev. Colomb. Obst. Gin. 2: 83, 1951.

16 COPPELSON. Citado por Rodríguez-Velasco en "Enfermedad Trofoblástica". Mem. III Cong. Peruano Obst. Ginec. 1: 443, 1968.

17 DEMERS, F. X. Moles hydatiformes. L'Union Méd. Canada. 84: 1397, 1958.

18 DE SNOO, K. Citado por Smalbraak en "Trophoblastic growths". Elsevier Press. 1957.

19 DEL CORRAL, F., ARTUZ, A., GHITIS, J. Y TAKANO, J. Efecto del Methoretxate sobre el coriocarcinoma secundario. Rev. Colomb. Obst. Gin. 13: 89, 1962.

20 DUARTE-CONTRERAS, A., ZUÑIGA-GAMBOA, R. y PALAU-C. M. J. E7I Urocitograma en Obstetricia. Rev. Colomb. Obst. Gin. 17: 195, 1966.

21 ENGELHARDT. Citado por Nubiola, P. y Zárate, E. en Tratado de Obstetricia. Ed. Laobr, Barcelona. 2: 634, 1951.

22 ESTRADA-C., A. Mola hidaitforme. Tesis de grado. Universidad de Antioquia, Fac. de Med. Inédita. 1928.

23 ESTRADA-OCAMPO, G. Eclampsia y preeclampsia coincidiendo con embarazo molar. Cinco Casos. Ginec. Obstet. Méx. 13: $359,1959$.

24 FERNANDEZ M. e Marques, C. Citado por Márquez-Monter y col. 38 .

25 FERNANDEZ-BASTIDAS, M. A. Corioepiteliama. Rev. Colomb. Obst. Gin. 5: 135, 1953.

26 FERNANDEZ-DOBLADO, R. Mola hidatiforme. Experiencias en el Hosjital Central Militar. Mem. III Cong. Mex. y Lat. Amer. Ginec. Obstet. 2: 570, 1958.
Rev. Colomb. Obst. Gin. 8: 371, 1957.

28 HASEGAWA. Citado por Rodríguez Velasco en "Enfermedad trofoblástica". Mem. III Cong. Peruano Obst. Gin. 1: 443, 1968.

29 HERTIG, A. T. and SHELDOM, W. H. Hydatiform mole: a pathologic-clinical correlation of 200 cases. Am. J. Obst. Gynec. 53 : $1,1947$.

30 HERTIG, A. T. and MANSELL, H. Hydatidiform mole and Choriocarcinoma. In "Atlas of Tumor Pathology". Part I, Fasc. 3, Sect. IV. Armed Forces Institute of Pathology, Washington, D. C., 1956.

31 JefFCOATE, T. N. A. Principes of Gynecology. Butterworth, London. 213, 1957.

32 JOURAVIEFF, A. Mole hydatiforme et chorioépitheliome. Gin. et Obst. 27: 223, 1933.

33 JORDAN-A., G. y LOPEZ-ESCOBAR, G. Corioepitelioma. Revisión de casos del Instituto Nacional de Cancerología. Rev. Colom. Obst. Gin. 10: 34, 1959.

34 KARZAVINA, E. G. Citado por MárquezMonter y col. 38.

35 KING, G. Citado por McGregor. 39.

36 LENCIONI, L. J., MARTINEZ AMEZAGA, L. A., LOBIANCO, V. S., OBERTI, M. R. y ALONSO, C. EI Urocitograma en el embarazo normal y patológico. Rev. Colomb. Obs. Gin. 15: 451, 1964.

37 LOPEZ-VALENCIA, R. Mola hidatiforme y su estadística (años 1934 a 1944) inclusive). Tesis de grado. Un. Antioquia. Fac. Med. Inédita. 1945.

38 MARQUEZ-MONTER, R., ALFARO DE LA VEGA, G., ROBLES, $M$. and BOLIO-CICERO, A. Epidemiology and Pathology of hydatidiform mole in the General Hospital of Mexico. Study of 104 cases. Am. J. Obst. Gynec. 85: 856, 1963.

39 MCGREGOR, C., ONTIVEROS-C., M., VARGAS-L., E. and VALENZUELA L. Hydatidiform mole. Analysis of 145 patients. Obst. and Gynec. 33: 343, 1969.

40 MERA-GONZALEZ, F. Degeneración corioepitelial después de un embarazo a término. Rev. Colomb. Obst. Gin. 3: 91, 1952.

41 MEYER, R. R. Citado por Nubiola, P. y Zárate, E. En "Tratado de Obstetricia". Ed. Labor, Barcelona. 2: 591, 1951. 
42 NOVAK, E. R. y WOODRUFF, J. D. Mola hidatiforme y corioepitelioma maligno. En "Ginecología y Obstetricia". Ed. Alhambra, S. A. Madrid. 2a. Ed. 582, 1964.

43 OROZCO RIQUETT, F. S. Tumores malignos del cuerpo uterino y coriocarcinoma. Rev. Colomb. Obst. Gin. 13: 195, 1962.

44 PARSON, L. and SOMERS, S. Gynencology. Ed. W. B. Saunders, Philadeljphia. 408, 1963.

45 PERNIA-PEREZ, A. Mola hidatiforme en los Hospitales Vargas y Central de San Cristóbal. Rev. Venez. Obst. Gin. 21: 243, 1961.

46 PINEDO, G. Mola hidatiforme y su evolución. Rev. Venez. Obst. Gin. 20: 51, 1960.

47 POEN, J. T. and DJOJOPRANOTO, M. The possible etiologic factor of hydatidiform $m-0$ le and choriocarcinoma. Am. J. Obst. Gynec. 92: 560,1965

48 ROSILLO-GALLARDO, J. A. Consideraciones generales sobre la mola hidatiforme y corioepitelioma. Tesis de grado. Un. de Cartagena. Fac. Med. y Cienc. Naturales. Inédita, 195? (en mimeógrafo en Biblioteca U. Antioquia).

49 SANCHEZ-TORRES, F., SANTAMARIA, A., ROCHA, A. and GOMEZ J. A. Renal changes in hydatidiform mole with toxemia. Am. J. Obst. Gynec. 92: 498, 1965.

50 SCHUMANN, E. A. and VOEGELIN, A. W. Corioepithelioma with special reference to its relative frequency. Am. J. Obst. Gynec. 33: 473, 1937.

51 SOBERON, A. J. y cols. Estudio clínico patológico de 7 casos de coriocarcinoma. Ginec. Obst. Méx. 22: 857, 1967.

52 USUBIAGA, I. Citado por Lencioni en "EI Urccitograma'^. Ed. Panamericana, Buenos Aires. 165, 1963.

53 VALENCIA HURTADO, F., GONGORA SANCHEZ, H., VELEZ RAMIREZ, O., MEJIA VIEIRA, F. Y MEJIA ROJAS E. Mola hidatiforme. Revisión de 91 casos clínicos en el Hospital Universitario. Unidad Obstétrica. Manizales. Rev. Colomb. Obst. Gin. 15: $345,1964$.

54 VERGARA-TAMARA, R. y RAMIREZ, H. Tumores trofoblásticos. Rev. Colomb. Obst. Ginec. 18: 165, 1967

55 VERGARA-TAMARA, R. Un caso de mola hidatiforme. Universitas Méd. 2: 77, 1954.

56 VILLAVICENCIO. Citado por Rodríguez Velasco en "Enfermedad Trofoblástica". Mem. III Cong. Peruano Obst. Gin. 1: 443, 1968.

57 WEI-PING, Y. and OUYANG, P. C. Trophoblastic diseases in Taiwan. Am. J. Obst. Gynec. 85: 844, 1963.

58 WEATERHOUT. F. C., MOREL, E. S. annd SLATE, W. C. Observation of 138 molar pregnancies. Am. J. Obst. Gynec. 103: 56, 1969.

59 ZABALETA-LOMBANA, A. Y MILANES PETNET, J. Mola hidatiforme benigna. Rev. Colomb. Obst. Gin. 16: 459, 1965. 